\title{
Invasive Lobular Breast Carcinoma, Pleomorphic Variant
}

National Cancer Institute

\section{Source}

National Cancer Institute. Invasive Lobular Breast Carcinoma, Pleomorphic Variant. NCI

Thesaurus. Code 697051.

A grade II invasive lobular carcinoma of the breast, characterized by the presence of neoplastic cells with large and atypical nuclei. 